will be found to conform with precise mathematical accuracy to any law : all the conclusions of biology are approximations.

\title{
REFERENCES
}

Duke-Elder, W. S. (1926).-Brit. Jl. of Ophthal., Vol. X, p. 1.

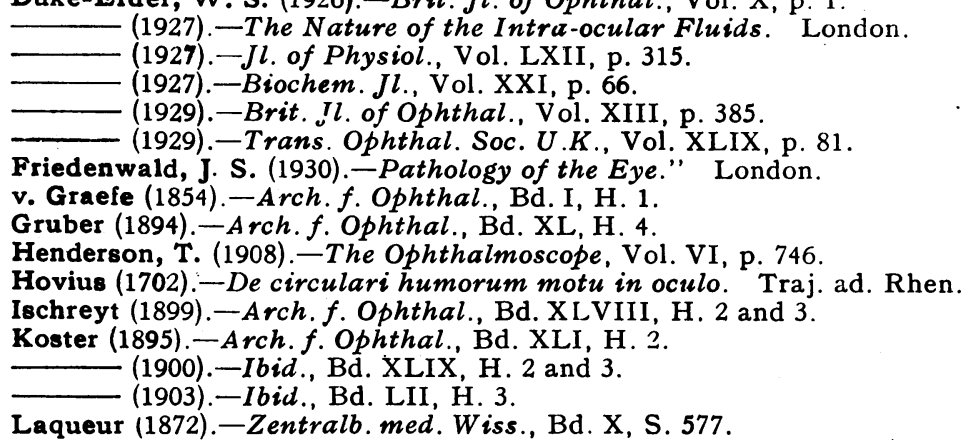

Laqueur (1872).-Zentralb. med. Wiss., Bd. X, S. 577.

Leber, T. H. (1899).-Arch.f. Ophthal., Bd. XXXV, H. 1.

(1903).-Graefe-Saemisch Handb., II Aufl., Bd. I, Teil 2.

Maggiore (1917).-Struttura, comportamento e significato del Canale di Schlemm nell' occhio umano. Rome.

Magitot, A. (1917).-Ann. d'Ocul., T. CLIV, pp. 272, 334, 385.

Nuel and Benoit (1895).-Arch. f. Ophthal., Bd. XLI.

Parsons, J. H. (1904). - Pathology of the Eye. London.

de Ruiter (1853).-Inaug. Dissertation. Traj. ad. Rhen.

Thomson, A. (1911). - The Ophthalmoscope, Vol. IX, p. 470.

Weber (1876).-Arch.f. Ophthal., Bd. XXIII, H. 1.

\section{TORIC SPECTACLES}

BY

\section{A. S. Percival, M.B.}

THE outer rim of an inflated motor tyre tube presents a toric surface; on transverse section a small circle would be seen, but on longitudinal section the outer rim would be represented by a much larger circle of much less curvature. A small area of this tube would present the toric form of a convex spherocylinder where its two principal powers are worked on the same surface with their axes at right angles to one another. A convex toric very roughly resembles the side of an egg seen from the outside, and a concave toric the side of an egg seen from the inside, but an egg is only circular in transverse section.

Toric tools are made to bases of $\pm 3.0 \mathrm{D} ., \pm 6.0 \mathrm{D}$. and $+9.0 \mathrm{D}$., the base $\pm 6.0 \mathrm{D}$. being the commonest, although for exact periscopic work many others are needed. The base (B) is always the lower power of the cylinder required, in this paper the higher power will be represented by $\mathbf{B}^{\prime}$.

A toric lens should be prescribed in the form of crossed cylinders, or if not, the toric base should always be specified. 
For instance a spherocylinder $+3 \cdot 0 \mathrm{D}$. sph. $+2 \cdot 0 \mathrm{D}$. cyl. ax. $90^{\circ}$, if prescribed as a toric on a $+3 \cdot 0 \mathrm{D}$. base, is written $+3 \cdot 0 \mathrm{D}$. cyl. ax. $180^{\circ}+5 \cdot 0$ D. cyl. ax. $90^{\circ}$.

To express any spherocylinder as a toric lens combination.

Write the spherocylinder in the form of crossed cylinders.

Three cases may arise :-

I. Crossed cylinders of the same sign.

Let $\mathrm{C}_{1}$ denote the lower, $\mathrm{C}_{2}$ the higher cylinder.

(1) Base B of the same sign as that of the crossed cylinders. Let $C_{1}-B=S$ where $S$ is the new spherical surface $\therefore \mathrm{C}_{1}-\mathrm{S}=\mathrm{B}$ and $\mathrm{C}_{2}-\mathrm{S}=\mathrm{B}^{\prime}$.

(2) Base B of opposite sign to that of the crossed cylinders. Let $\mathrm{C}_{2}-\mathrm{B}=\mathrm{S}$ $\therefore \mathrm{C}_{2}-\mathrm{S}=\mathrm{B}$ and $\mathrm{C}_{1}-\mathrm{S}=\mathrm{B}^{\prime}$.

II. Crossed cylinders of opposite sign.

Let $\mathrm{C}$ be that cylinder whose sign is opposite to that of $\mathrm{B}$, then $\mathrm{C}-\mathrm{B}=\mathrm{S}$.

Examples I. (1) +2.5 D. sph. +1.5 D. cyl. ax. $80^{\circ}$ written as crossed cyls. $+25^{\circ}$ D. cyl. ax. $170^{\circ}+4 \cdot 0$ D. cyl. ax. $80^{\circ}$.

Suppose the base $+3 \cdot 0 \mathrm{D}$. be chosen, then

2.5 D. -3.0 D. $=\mathrm{S}=-0.5 \mathrm{D}$. sph.

$\mathrm{C}_{1}-\mathrm{S}=\mathrm{B}$ or $2 \cdot 5+0 \cdot 5=+3 \cdot 0 \mathrm{D}$.

$\mathrm{C}_{2}-\mathrm{S}=\mathrm{B}^{\prime}$ or $4+0 \cdot 5=+4 \cdot 5 \mathrm{D}$.

and the prescription is $+3 \cdot 0$ D. cyl. ax. $170^{\circ}+4 \cdot 5$ D. cyl. ax. $80^{\circ}$ $-0.5 \mathrm{D}$.

This means that the toric surface is to be placed anteriorly and the concave surface is to be placed next the eye.

I. (2) Suppose that we wish to prescribe the same spherocylinder in toric form on a $-6.0 \mathrm{D}$. base.

4 D. +6 D. $:=\mathrm{S}=+10^{\circ} 0$ D. sph.

$\mathrm{C}_{2}-\mathrm{S}=4-10=-6.0$ D.; $\mathrm{C}_{1}-\mathrm{S}=2.5-10=-7.5 \mathrm{D}$. so the prescription is $+10.0 \mathrm{D}$.

$$
-6.0 \text { D. cyl. ax. } 80^{\circ}-7 \cdot 5 \text { D. cyl. ax. } 170^{\circ}
$$

II. Spherocylinder -1.0 D. sph. $+3 \cdot 0$ D. cyl. ax. $100^{\circ}$

Crossed cyls. $-1 \cdot 0$ D. cyl. ax. $10^{\circ}+2 \cdot 0$ D. cyl. ax. $100^{\circ}$

Let base be $+6 \cdot 0 \mathrm{D}$.

$-1.0 \mathrm{D} .-6.0 \mathrm{D} .=\mathrm{S}=-7.0 \mathrm{D} . \mathrm{sph}$.

$-1 \cdot 0 \mathrm{D} .+7 \cdot 0 \mathrm{D} .=+6 \cdot 0 \mathrm{D} . ;+2 \cdot 0 \mathrm{D} .+7 \cdot 0 \mathrm{D} .=+9 \cdot 0 \mathrm{D}$.

$$
\frac{+6 \cdot 0 \text { D. cyl. ax. } 10^{\circ}+9 \cdot 0 \text { D. cyl. ax. } 100^{\circ}}{-7 \cdot 0 \text { D. }}
$$

is the prescription for the toric combination.

A patient of mine once showed me a prescription that was given to her by another specialist in the form $+2.5 \mathrm{D} . \mathrm{sph} .+1.5 \mathrm{D}$. cyl. ax. $80^{\circ}$ with the note at the bottom " give torics." It is our 
duty to order those torics that will be of real service if we order them at all. In the example given in I (1) there would be little advantage over the ordinary spherocylinder provided that the cylindrical surface were ordered to be next the eye.

It is clear on referring to the table at the end of this paper that in the meridian at $80^{\circ}$, where the power is $+2.5 \mathrm{D}$., the ocular concavity should be $-6.5 \mathrm{D}$.; while in the meridian at $170^{\circ}$, where the power is $+4.0 \mathrm{D}$., the ocular concavity should be $-6.75 \mathrm{D}$.

Again in the second example, although it is better than the first, where the power is $2.5 \mathrm{D}$. the concavity is $-6.0 \mathrm{D}$., and where the power is $+4.0 \mathrm{D}$. the concavity is $-7.5 \mathrm{D}$.

There are six different ways in which the optician could have obeyed the orders of the ophthalmic surgeon in giving toric lenses to this patient, but only one of them would give really satisfactory results with the tools in use namely that on a $+9^{\circ} 0 \mathrm{D}$. base. It is our business, if we order toric lenses, to state what kind of toric lenses we wish; just as much as it is a physician's business to give the quantities of the medicines he prescribes.

The best prescription for this spherocylinder that will give the greatest range of vision, when the eyes are rotated behind it, is

$$
\frac{+9.0 \text { D. cyl. ax } 470^{\circ}+10.5 \text { D. cyl. ax. } 80^{\circ}}{-6.5 \text { D. sph. }}
$$

The last example for $-1.0 \mathrm{D}$. sph. $+3.0 \mathrm{D}$. cyl. ax. $100^{\circ}$ is not very good, but it is the best that can be obtained with the ordinary tools. The concavity given is $-7 \cdot 0 \mathrm{D}$, it should be $-7 \cdot 25 \mathrm{D}$. in one meridian and $-6.5 \mathrm{D}$. in the other meridian. Theoretically, the best prescription for. periscopic purposes would be \begin{tabular}{l}
$+6 \cdot 25$ D. cyl. ax. $10^{\circ}+8 \cdot 5$ D. cyl. ax. $100^{\circ}$ \\
\hline$-6 \cdot 5$ D. cyl. ax. $100^{\circ}-7 \cdot 25$ D. cyl. ax. $10^{\circ}$
\end{tabular}

but tools are not made for either of these torics. Practically nearly all patients would be quite satisfied with the ordinary spherocylinder if the $-1.0 \mathrm{D}$. sph. were placed next the eye.

In conclusion we may say that for low powers of concave spherocylinders toric lenses are almost unnecessary, provided that the concave spherical surface is placed next the eye. Frequently a transposition of the cylinders may be advisable, if that will render the ocular surface more nearly that recommended in the table.

For instance, -3.5 D. sph. -3.0 D. cyl. ax. $180^{\circ}$ if transposed to $-6.5 \mathrm{D}$. sph. $+3.0 \mathrm{D}$. cyl. ax. $90^{\circ}$ would be an almost perfect periscopic reading glass, and would allow a very wide field for distance. It is true that the glasses would be slightly heavier than those in the ordinary form, but the periscopic advantage would more than compensate for that objection. 
It will be noticed that in the case of concave spherocylinders a different ocular curvature is recommended for each power in the two meridians of the crossed cylinders, so that in most cases two torics made by special tools for each lens would be required, which would render the price prohibitive.

Whenever high convex glasses are required there is a much more urgent call for periscopic glasses than in cases of myopia, for the field of view on oblique vision through biconvex lenses is much more restricted than through biconcave lenses. However, fortunately the ocular concavity varies for distant vision only between $-6.5 \mathrm{D}$. and $-\tau \cdot 0 \mathrm{D}$. between the powers of $1.0 \mathrm{D}$. and $+12.5 \mathrm{D}$., so it would appear that all convex spherocylinders for distance should be ordered with the ocular surface having a concavity of $-6.5 \mathrm{D}$. to $-7.0 \mathrm{D}$. sph. But as $+9.0 \mathrm{D}$. base is the highest toric made, we are restricted from dealing in this way with any convex spherocylinder whose lower axis is more than about $+2.5 \mathrm{D}$. We are obliged in such cases as $+10.0 \mathrm{D}$. sph. $+2.0 \mathrm{D}$. cyl. ax. $180^{\circ}$, as may be required in aphakia, to make the ocular surface a toric one on base -6.0 D.; e.g., $+18.0 \mathrm{D}$.

$$
-6^{\circ} 0 \text { D. cyl. ax. } 180^{\circ}-8 \cdot 0 \text { D. cyl. ax. } 90^{\circ}
$$

The ocular surface is, of course, not correct but it is the best that can be made with the present tools; there is, however, a far graver error in the prescription which is inexcusable because it can be rectified.

The thickness of the meniscus is roughly given by Mr. Laurance's simple formula for a $40 \mathrm{~mm}$. lens : $\mathrm{t}=1+0.4$ (D) $=$ $1+4.8 \mathrm{~mm}$. ' $=5.8 \mathrm{~mm}$.

Where $\mathrm{D}=12$ in the vertical axis the power of the lens is $+12 \cdot 41 \mathrm{D}$. as if placed $5.5 \mathrm{~mm}$. in front of its true position, its effectivity is that of a lens of power $+13 \cdot 32$ D. placed $13.6 \mathrm{~mm}$. from the cornea. The anterior surface of the meniscus is far too convex; the patient would not only fail to see distinctly but he would complain of a distressing curvature of the field. The true anterior curvature, as given in my book, p. 73, is the value of $d_{1}$, where $d_{1}=\frac{\mu\left(D_{b}-d_{2}\right)}{\mu+\left(D_{b}-d_{2}\right) t}$. Here we may take the effective power of the required lens $\left(D_{b}\right)$ placed $13.6 \mathrm{~mm}$. from the cornea, i.e., at the back surface of the lens) as $+11^{\circ} 0 \mathrm{D}$., as it is the mean between $+10.0 \mathrm{D}$. and $+12.0 \mathrm{D}$.; and similarly $-\mathrm{d}_{2}$ as 7.0 D. The thickness $t$ is $0.0058 \mathrm{~m}$. for the unit in dioptric formulae must be metric. We have then

$$
\mathrm{d}_{1}=\frac{1 \cdot 523(18)}{1 \cdot 523+18(0 \cdot 0058)}=\frac{2 \tau \cdot 414}{1 \cdot 6274}=16 \cdot 84 .
$$

Practically, it is always better to give a glass that is a trifle weaker, 
than a trifle stronger, than is necessary to avoid curvature of the field, so the prescription should be written

$$
\frac{+16.75 \text { D. sph. }}{-6.0 \text { D. cyl. ax. } 180^{\circ}-8.0 \text { D. cyl. ax. } 90^{\circ}}
$$

This meniscus would have practically the effectivity of the required spherocylinder $+10^{\circ} 0$ D. sph. $+2 \cdot 0$ D. cyl. ax. $180^{\circ}$ This example will show the great importance of the thickness of the lens in high powered convex menisci. A glance at the table will indicate roughly the power required for the convex surface without this tedious calculation. A periscopic of $+11^{\circ} 0$ on a $-6.75 \mathrm{D}$. base requires a $+16.697 \mathrm{D}$. anterior surface, so on a -7.0 D. base will require roughly a $+16.697+0.25$ or a +16.847 D. anterior surface. Indeed, unless the patientcan wear the glasses very close to his eyes the previous prescriptions should be made $0.25 \mathrm{D}$. weaker; so that even the length of the eyelashes must be considered for the really accurate prescription of periscopic menisci.

After a cataract operation there is almost always a certain amount of induced astigmatism, requiring a positive cylinder with its axis horizontal; in the course of two or three months this astigmatism usually diminishes. Suppose that +10.0 D. sph. $+20^{\circ}$ D. cyl. ax. $180^{\circ}$ is required. It saves expense if a biconvex $+9.5 \mathrm{I}$ ). is ordered to be tilted downwards about $23^{\circ}$. On referring to the little table, p. 69 of my book, it will be seen that $25^{\circ}$ is too great and $20^{\circ}$ is too small an angle. It is unnecessary to find the angle accurately as when the glasses are worn the patient will look through them at varying angles. I have always adopted this device for the first temporary glasses, and after five or six months, when the eyes have settled down, ordered the permanent glasses. If there be no astigmatism, it is a great help to order a meniscus with a concavity of $-4.5 \mathrm{D}$. or so for reading purposes : they will be able to read without moving their head for every line. In such cases bifocals are of great advantage, when carefully fitted. The ocular surface for distance will be about $-6.75 \mathrm{D}$. or $-7 \cdot 0$ curvature, and if the reading wafer be cemented to the lower part of the concave surface but does not extend to its lower edge, the patient will have a fair periscopic field for reading, and will still be able to see steps through the lower edge when going up or down stairs.

Bifocals are unsatisfactory for aphakic patients who require a cylindrical correction, as the reading wafer must be attached to the convex surface, which makes the meniscus of the wrong shape for reading. As the object of a periscopic lens is to enable one to see distinctly when looking obliquely through it, tiling a periscopic lens will not correct astigmatism against the rule, though I have heard this suggestion made. 
As expressed elsewhere I do not recommend bifocals for myopes unless of very low degree. In two years I had two patients with a myopia of less than $-6.0 \mathrm{D}$. who acquired a detached retina after wearing bifocal lenses, one pair having been prescribed by myself.

\section{CONVEX PERISCOPIC LENSES OF WIDTH $40 \mathrm{~mm}$.}

Solid angle of $60^{\circ}, \mu=1^{\circ} 523$.

Distance.

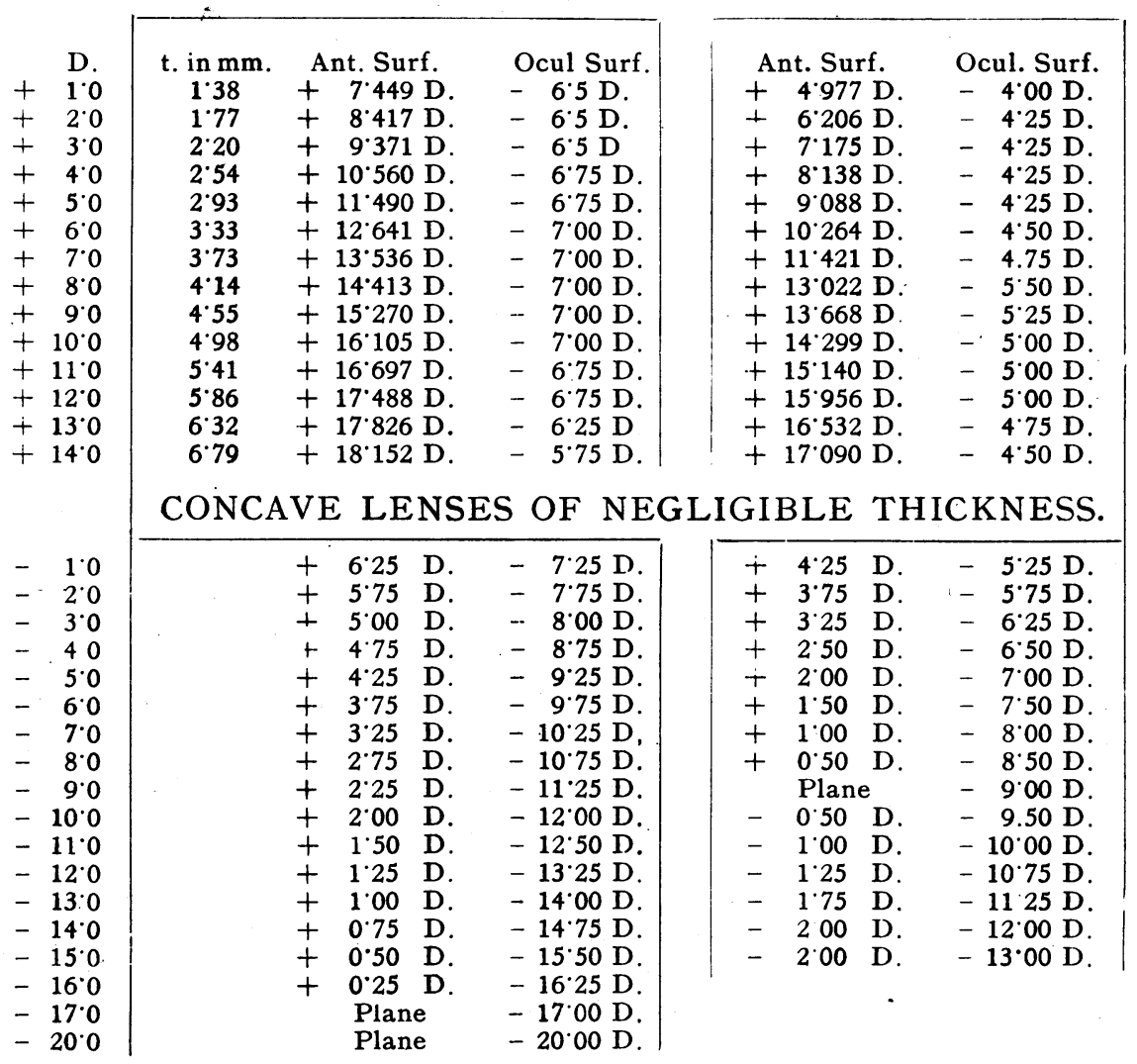

